PROCEEDINGS OF THE

AMERICAN MATHEMATICAL SOCIETY

Volume 132, Number 8 , Pages $2465-2474$

S 0002-9939(04)07196-5

Article electronically published on March 24, 2004

\title{
DIFFUSIONS, EXIT TIME MOMENTS AND WEIERSTRASS THEOREMS
}

\author{
VICTOR H. DE LA PEÑA AND PATRICK MCDONALD
}

(Communicated by Richard C. Bradley)

\begin{abstract}
Let $X_{t}$ be a one-dimensional diffusion with infinitesimal generator given by the operator $L=\frac{1}{2}\left(a(x) \frac{d}{d x}\right)^{2}+b(x) \frac{d}{d x}$ where $a(x)$ is a smooth, positive real-valued function and the ratio of $a(x)$ and $b(x)$ is a constant. Given a compact interval, we prove a Weierstrass-type theorem for the exit time moments of $X_{t}$ and their corresponding (naturally weighted) first derivatives, and we provide an algorithm that produces uniform approximations of arbitrary continuous functions by exit time moments. We investigate analogues of these results in higher-dimensional Euclidean spaces. We give expansions for several families of special functions in terms of exit time moments.
\end{abstract}

\section{INTRODUCTION}

The purpose of this note is to develop a natural link between Brownian motions and approximation theory. Our results establish that, in one dimension, continuous functions can be well approximated by exit time moments of general Brownian motions and their naturally weighted first derivatives, and we provide a natural algorithm that produces uniform approximations to arbitrary continuous functions using exit time moments. We establish related statements in higher-dimensional Euclidean space. To concisely state our results, we begin by introducing some notation.

Let $a: \mathbb{R} \rightarrow \mathbb{R}$ be a smooth (infinitely differentiable) positive function and let $g(x)=\frac{1}{a^{2}(x)}$. Then $g$ defines a (Riemannian) metric on the line with corresponding Laplace operator $\left(a(x) \frac{d}{d x}\right)^{2}$. Let $X_{t}$ be Brownian motion on the line with respect to the metric $g$. Thus, $X_{t}$ is a diffusion on $\mathbb{R}$ with infinitesimal generator $\mathcal{L}=$ $\frac{1}{2}\left(a(x) \frac{d}{d x}\right)^{2}$. Let $\mathbb{P}^{x}, x \in \mathbb{R}$ be the associated family of probability measures charging paths beginning at the point $x$.

Let $I=[\alpha, \beta]$ be any compact interval and let $\tau$ be the first exit time of $X_{t}$ from $I$ :

$$
\tau=\inf \left\{t \geq 0: X_{t} \notin I\right\}
$$

Received by the editors August 13, 2002.

2000 Mathematics Subject Classification. Primary 60J65, 40A30.

Key words and phrases. Brownian motion, exit time moments, approximation theory, Bessel functions.

(C)2004 American Mathematical Society 
Let $\gamma$ be the constant

$$
\gamma=(\beta-\alpha)\left(\int_{\alpha}^{\beta} \frac{1}{a(s)} d s\right)^{-1} .
$$

For each natural number $k$, let $v_{k}: I \rightarrow \mathbb{R}$ be the $k$ th moment of $\tau$ :

$$
v_{k}(x)=\mathbb{E}^{x}\left[\tau^{k}\right]
$$

where $\mathbb{E}^{x}$ is expectation with respect to the measure $\mathbb{P}^{x}$. For $k=0$ set

$$
v_{0}(x)=1
$$

and for $k$ a negative integer set

$$
v_{k}(x)=\gamma^{-1} a(x) \frac{d}{d x} v_{|k|}(x) .
$$

Our main result, a Weierstrass-type theorem, is that any continuous function on $I$ can be uniformly approximated by linear combinations of the $v_{k}$. More precisely,

Theorem 1.1. Let $X_{t}$ be a diffusion on $\mathbb{R}$ with infinitesimal generator $\mathcal{L}=\frac{1}{2}\left(a \frac{d}{d x}\right)^{2}$, a uniformly elliptic operator with smooth coefficients. Let $I=[\alpha, \beta]$ be any compact interval, let $\tau$ be the first exit time of $X_{t}$ from $I$, and, for $k$ an integer, let $v_{k}(x)$ be defined by (1.2)-(1.4). Then linear combinations of the elements $v_{k}, k$ an integer, are uniformly dense in the space of continuous functions on $I$.

Using the same techniques as those used to establish Theorem 1.1 we provide a proof that the conclusion of Theorem 1.1 holds for classical Brownian motion with drift. This leads immediately to the corresponding result for a one-dimensional diffusion process, $X_{t}$, given as the solution of the stochastic differential equation

$$
d X_{t}=a\left(X_{t}\right) d W_{t}+a\left(X_{t}\right)\left(b+a^{\prime}\left(X_{t}\right)\right) d t
$$

where $W_{t}$ is standard Brownian motion, $a(x)$ is a smooth positive real-valued function and $b$ is a constant (equivalently, the infinitesimal generator of $X_{t}$ is $\mathcal{L}=\frac{1}{2}\left(a(x) \frac{d}{d x}\right)^{2}+b a(x) \frac{d}{d x} ;$ cf. Theorem 3.2). New techniques will be required to further generalize these results.

We note that the most direct generalization of our results to higher dimensions is clearly false. In fact, if $B$ is a ball in $\mathbb{R}^{n}$, then the exit time moments of Brownian motion are radial functions, as are all derivatives (in particular, all angular dependence is lost). This example suggests that we consider the moments of Brownian motion restricted to certain one-dimensional subsets. We provide results in this direction: If $B$ is a ball in $\mathbb{R}^{n}$, then the exit time moments of standard Brownian motion restricted to any line through the center of the ball, together with the corresponding first derivatives, have a dense linear span in the space of continuous functions on the line (cf. Theorem 4.1).

Understanding the properties that lead to best approximations and the corresponding representation theory is a natural, intuitively appealing problem. This problem is not as straightforward as one might hope in that the exit time moments are not orthogonal with respect to any positive measure. Nonetheless, we establish a natural algorithm which produces uniform approximations of arbitrary continuous functions in terms of exit time moments (cf. Theorem [5.2). We also provide explicit expansions of certain special functions (e.g. Bessel functions) in terms of exit time moments. 


\section{EXIT TIME MOMENTS AND BOUNDARY VALUE PROBLEMS}

In this section we collect a number of facts concerning the relationship between exit time moments and boundary value problems which we will use in the sequel. The material is by no means new (cf. [Ha], $[\mathrm{KMM}]$ ) and is included for clarity of exposition.

Let $X_{t}$ be a diffusion on $\mathbb{R}^{n}$ with associated probabilities $\mathbb{P}^{x}, x \in \mathbb{R}^{n}$, charging paths beginning at $x$. Let $\mathbb{E}^{x}$ be the corresponding expectation operators. Suppose that $X_{t}$ has infinitesimal generator $\mathcal{L}$, a second-order uniformly elliptic operator with smooth coefficients. Let $D$ be a smoothly bounded domain with compact closure and let $\tau$ be the first exit time of $X_{t}$ from $D$ :

$$
\tau=\inf \left\{t \geq 0: X_{t} \notin D\right\} .
$$

It is well known that the distribution of $\tau, u(x, t)=\mathbb{P}^{x}(\tau>t)$, gives the solution of the initial value problem:

$$
\begin{aligned}
\frac{\partial u}{\partial t} & =\mathcal{L} u \text { on } D \times(0, \infty), \\
u(x, 0) & = \begin{cases}1 & \text { if } x \in D, \\
0 & \text { if } x \in \partial D,\end{cases} \\
u(x, t) & =0 \text { on } \partial D \times(0, \infty) .
\end{aligned}
$$

Let $h(x, s)$ denote the Laplace transform of $\tau$ :

$$
h(x, s)=\mathbb{E}^{x}\left[e^{-s \tau}\right] .
$$

Then for $s>0$ fixed, $h$ is the unique solution of the Dirichlet problem

$$
\begin{aligned}
\mathcal{L} h-s h & =0 \text { on } D, \\
h & =1 \text { on } \partial D .
\end{aligned}
$$

Using the power series for the exponential function, we have

$$
h(x, s)=\sum_{k=0}^{\infty} \mathbb{E}^{x}\left[\tau^{k}\right] \frac{(-s)^{k}}{k !} .
$$

Starting with (2.3) and (2.4) and equating coefficients, we conclude that if $u_{k}$ is defined inductively by

$$
\begin{aligned}
\mathcal{L} u_{1}+1 & =0 \text { on } D, \\
u_{1} & =0 \text { on } \partial D,
\end{aligned}
$$

and if

$$
\begin{aligned}
\mathcal{L} u_{k}+k u_{k-1} & =0 \text { on } D, \\
u_{k} & =0 \text { on } \partial D,
\end{aligned}
$$

then

$$
u_{k}(x)=\mathbb{E}^{x}\left[\tau^{k}\right]
$$




\section{Results in one Dimension}

Proof of Theorem [1.1. Suppose that $X_{t}$ is standard Brownian motion on the line and denote by $\mathcal{L}=\frac{1}{2} \frac{d^{2}}{d x^{2}}$ the corresponding infinitesimal generator of $X_{t}$. Let $I=$ $(\alpha, \beta)$ and recall that the exit time moments of $X_{t}$ are defined recursively by (2.5)(2.6) in terms of the solutions of Poisson problems on $I(=D)$. Setting $\mu=\frac{\alpha+\beta}{2}$ and choosing coordinates at $\mu$, an easy induction then shows that the moments of the exit time are polynomials symmetric about $\mu$ of the form

$$
u_{k}(x)=\sum_{j=0}^{k} c_{k, 2 j} x^{2 j}
$$

Setting $\delta=\frac{\beta-\alpha}{2}$, the coefficients $c_{k, 2 j}$ are defined by recursion:

$$
u_{1}(x)=\delta^{2}-x^{2}
$$

and if $u_{k}$ is given by (3.1), then $u_{k+1}$ is given by

$$
u_{k+1}(x)=-2(k+1) \sum_{j=0}^{k} \frac{c_{k, 2 j}}{(2 j+1)(2 j+2)} x^{2(j+1)}+\gamma_{1} x+\gamma_{0} .
$$

Since $u_{k+1}^{\prime}(0)=0, \gamma_{1}=0$ and the coefficients of $u_{k+1}$ are given by

$$
c_{k+1,2 j}= \begin{cases}-2(k+1) \frac{c_{k, 2(j-1)}}{(2 j-1)(2 j)} & \text { if } j>0, \\ 2(k+1)\left(\sum_{i=0}^{k} \frac{c_{k, 2 i} \delta^{2(i+1)}}{(2 i+1)(2 i+2)}\right) & \text { if } j=0 .\end{cases}
$$

From (3.2) it follows that the constants are all nonzero. By observation, the derivatives of the moments,

$$
u_{-k}(x)=\sum_{j=1}^{k} 2 j c_{k, 2 j} x^{2 j-1}
$$

are all antisymmetric about $\mu$. Since none of the coefficients in the expressions of $u_{k}$ and $u_{-k}$ vanish, it follows that the collection of linear combinations of the $u_{k}$ for $k$ an integer is the space of polynomials in $x$. By Weierstrass' theorem, the theorem follows for the special case when $X_{t}$ is classical Brownian motion.

Before proceeding to the case of more general diffusions, we note that the constants $c_{k, 2 j}$ have natural probabilistic descriptions:

$$
\begin{aligned}
c_{k, 0} & =\sup _{x \in I} \mathbb{E}^{x}\left[\tau^{k}\right], \\
c_{k, 2 j} & =\frac{(-2)^{j}(k !)}{(2 j) !(k-j) !} \sup _{x \in I} \mathbb{E}^{x}\left[\tau^{k-j}\right] .
\end{aligned}
$$

Suppose that $a(x)>0$ and that $\mathcal{L}=\frac{1}{2}\left(a(x) \frac{d}{d x}\right)^{2}$. Let $\gamma$ be the constant defined in (1.1) above. Let $\mathcal{M}: C([\alpha, \beta]) \rightarrow C([\alpha, \beta])$ be the change of variables map defined by

$$
\mathcal{M} f(x)=f\left(\alpha+\gamma \int_{\alpha}^{x} \frac{1}{a(s)} d s\right) .
$$

The transform $\mathcal{M}$ rescales the speed of the diffusion and is given by integration of the speed measure associated to the process $X_{t}$. Clearly, $\mathcal{M}$ is linear and invertible. 
Moreover, a simple computation gives the following (intertwining) relationship:

$$
a \frac{d}{d x}(\mathcal{M} f)=\gamma \mathcal{M}\left(\frac{d}{d x} f\right) .
$$

For $k$ an integer greater than zero, let $v_{k}$ be the solution to the Poisson hierarchy (2.5) -(2.6) above. Then if $\tau$ is the first exit time of $X_{t}$ from $I, \mathbb{E}^{x}\left[\tau^{k}\right]=v_{k}(x)$. For $k \leq 0$, let $v_{k}(x)$ be defined as in (1.3) and (1.4).

Let $k$ be positive. Using the relationship (3.6) we obtain

$$
\left(a \frac{d}{d x}\right)^{2} \mathcal{M} u_{k}=\gamma^{2}(-k) \mathcal{M} u_{k-1}
$$

Since $\mathcal{M} u_{k}(\alpha)=\mathcal{M} u_{k}(\beta)=0$, we conclude, by uniqueness of the solution of the Poisson problem, that

$$
\mathcal{M} u_{k}=\gamma^{2 k} v_{k}
$$

Finally, if $k<0$, we have

$$
\begin{aligned}
\mathcal{M} u_{k} & =\mathcal{M} u_{|k|}^{\prime} \\
& =\gamma^{-1} a \frac{d}{d x} \mathcal{M} u_{|k|} \\
& =\gamma^{2 k} \gamma^{-1} a \frac{d}{d x} v_{|k|}
\end{aligned}
$$

and thus, for all $k \neq 0,(3.7)$ holds. We conclude that $\left\{v_{k}\right\}$ is the image of a dense set under an invertible linear map. It follows that $\left\{v_{k}\right\}$ is dense in $C([\alpha, \beta])$, and the proof of the theorem is complete.

We note that the conclusion of Theorem 1.1 holds for processes obtained from Brownian motion by a wide class of transformations. As an example we consider the case of standard geometric Brownian motion (there is an analogous result for general geometric Brownian motions):

Corollary 3.1. Let $X_{t}$ be Brownian motion on the real line and let $\tilde{X}_{t}$ be the corresponding geometric Brownian motion. Let $\tilde{I}=[\tilde{\alpha}, \tilde{\beta}] \subset \mathbb{R}^{+}$be any compact interval in the positive real numbers and let $\tilde{\tau}$ be the first exit time of $\tilde{X}_{t}$ from $\tilde{I}$. For $k$ a nonnegative integer, let $\tilde{v}_{k}(x)$ be the exit time moments of $\tilde{X}_{t}$, and for $k$ a negative integer, let $v_{k}$ be the first derivative of $v_{|k|}$, weighted by $e^{x}$. Then linear combinations of the elements $\tilde{v}_{k}, k$ an integer, are uniformly dense in the space of continuous functions on $\tilde{I}$.

Proof. Let $\tilde{\alpha}=e^{\alpha}$ and $\tilde{\beta}=e^{\beta}$. Let $I=[\alpha, \beta]$, and let $\tau$ be the first exit time of $X_{t}$ from $I$. Then for all natural numbers $k$ and all $x$ in $I$,

$$
\mathbb{E}^{e^{x}}\left[\tilde{\tau}^{k}\right]=\mathbb{E}^{x}\left[\tau^{k}\right]
$$

The claim now follows from Theorem 1.1 and a change of variables.

We will use the techniques developed above to prove the following generalization of Theorem 1.1]

Theorem 3.2. Let $b$ be a constant and suppose that $a$ is a smooth positive function. Let $\mathcal{L}=\frac{1}{2}\left(a(x) \frac{d}{d x}\right)^{2}+b \gamma a(x) \frac{d}{d x}$ where $\gamma$ is the constant given by (1.1). Let $X_{t}$ be the diffusion process on the line with infinitesimal generator $\mathcal{L}$. Let $I=[\alpha, \beta]$ be a compact interval, and let $\tau$ be the first exit time of $X_{t}$ from $I$. For $k$ an integer, 
let $v_{k}$ be defined by (1.2)-(1.4). Then linear combinations of the $v_{k}$ are uniformly dense in the continuous functions on $I$.

Proof. Suppose that $a(x) \equiv 1$. Then the generator in question is given by

$$
\mathcal{L}=\frac{1}{2} \frac{d^{2}}{d x^{2}}+b \frac{d}{d x}
$$

and the process $X_{t}$ is classical Brownian motion with constant drift. The exit time moments for $X_{t}, \mathbb{E}^{x}\left[\tau^{k}\right]=u_{k}(x)$, are given by recursive solution of the Poisson problem (2.5)-(2.6). Introducing the integrating factor $w(x)=e^{2 b x}$ and noting that $\frac{d}{d x}\left(u_{k}^{\prime} w\right)=\left(u_{k}^{\prime \prime}+2 b u_{k}^{\prime}\right) w$, we see upon integrating twice that

$$
u_{k}(x)=\int_{\alpha}^{x} \frac{C_{k}-2 k \int_{\alpha}^{r} w(s) u_{k-1}(s) d s}{w(r)} d r
$$

where $C_{k}$ is chosen such that $u_{k}(\beta)=0$ (in particular, $C_{k} \neq 0$ for all $k$ ). A straightforward computation then gives

$$
u_{1}=E_{0} e^{-2 b x}+D_{1} x+D_{0}
$$

for nonzero constants $D_{0}, D_{1}$, and $E_{0}$. From this we conclude that

$$
\operatorname{span}\left\{u_{0}, u_{1}, u_{-1}\right\}=\operatorname{span}\left\{1, x, e^{-2 b x}\right\} .
$$

Assume that for all $j \leq k$,

$$
u_{j}(x)=\sum_{i=0}^{j} D_{j, i} x^{i}+\sum_{i=1}^{j} E_{j, i-1} x^{i-1} e^{-2 b x}
$$

where $D_{j, j}$ and $E_{j, j-1}$ are nonzero and that

$$
\operatorname{span}\left\{u_{j}: 0 \leq|j| \leq k\right\}=\operatorname{span}\left\{x^{l}, x^{j} e^{-2 b x}: 0 \leq l \leq k, 0 \leq j \leq k-1\right\} .
$$

An induction using (3.10) then gives that

$$
\operatorname{span}\left\{x^{j}: 0 \leq j \leq n\right\} \quad \subset \operatorname{span}\left\{u_{k}:|k| \leq n\right\},
$$

which proves the theorem in the case of classical Brownian motion with drift.

For the general case we reduce to classical Brownian motion with drift using the operator $\mathcal{M}: C(I) \rightarrow C(I)$ defined by (3.5). More precisely, suppose that $\mathcal{L}=\frac{1}{2}\left(a(x) \frac{d}{d x}\right)^{2}+b \gamma a(x) \frac{d}{d x}$, that $L$ is the operator given in (3.9) (the infinitesimal generator of Brownian motion with drift), and that $u_{k}(x)$ is as in (3.10). Then following exactly the same argument given in the proof of Theorem 1.1, we conclude that, for all $k \neq 0, \mathcal{M} u_{k}=\gamma^{2 k} v_{k}$. As was the case for Theorem [1.1] this proves Theorem 3.2.

\section{Higher Dimensions}

Let $X_{t}$ be Brownian motion on $\mathbb{R}^{n}$, and let $\mathcal{L}=\frac{1}{2} \Delta$ denote the infinitesimal generator of $X_{t}$. Let $B \subset \mathbb{R}^{n}$ be a ball of radius $\delta$, and let $\tau$ be the first exit time of $X_{t}$ from $B$. For $k=1$, the solutions of (2.6) are radial and can be given explicitly:

$$
u_{1}(|x|)=\frac{\delta^{2}-|x|^{2}}{n}
$$


where $|x|$ denotes the Euclidean distance from the center of the ball. From (2.6) and (4.1) it follows that the exit time moments of $X_{t}$ from $B$ are radial and given by the recursion

$$
u_{k}(|x|)=D_{k}-\int_{0}^{|x|} \frac{2 k \int_{0}^{q} s^{n-1} u_{k-1}(s) d s}{q^{n-1}} d q
$$

where the $D_{k}$ are chosen to satisfy the boundary conditions $u_{k}(\delta)=0$. Following the one-dimensional case, write

$$
u_{k}(|x|)=\sum_{j=0}^{k} d_{k, 2 j}|x|^{2 j} .
$$

The probabilistic definition of the coefficients $c_{k, 2 j}$ given in (3.2)-(3.3) generalizes to the $n$-dimensional case:

$$
\begin{aligned}
d_{k, 0} & =\sup _{x \in B} \mathbb{E}^{x}\left[\tau^{k}\right] \\
d_{k, 2 j} & =\frac{(-1)^{j} k !}{(k-j) ! j !} \frac{1}{\prod_{m=1}^{j}(2 j-(2 m-1)+(n-1))} \sup _{x \in B} \mathbb{E}^{x}\left[\tau^{k-j}\right] .
\end{aligned}
$$

There is an analogue of Theorem 1.1 .

Theorem 4.1. Let $X_{t}$ be standard Brownian motion on $\mathbb{R}^{n}$, and suppose that $B$ is a ball in $\mathbb{R}^{n}$. Let $l$ be the intersection of a line through $B$ with $B$. Let $u_{k}(x)$ be the kth moment of the exit time from $B$ of $X_{t}$, given that $X_{t}$ starts at $x \in l$ (that is, we restrict $u_{k}(x)$ to $\left.l\right)$. Let $u_{-k}$ be the directional derivative of $u_{k}$ along $l$ and suppose that $u_{0}(x)=1$. Then linear combinations of the elements $u_{k}, k$ an integer, are uniformly dense in the space of continuous functions on $l$.

Proof. Suppose that $l$ passes through the center of the ball. The expression (4.3) gives an explicit formula for the $u_{k}$ appearing in the hypotheses of the theorem. The proof of the theorem now follows from exactly the same argument as given in the proof of Theorem 1.1 in the case of Brownian motion.

When the line segment does not pass through the center of the ball, we can choose coordinates $\left(x_{1}, x_{2}, \ldots, x_{n}\right)$ such that the chord falls in the $\left(x_{1}, x_{2}\right)$-plane, along the line $x_{1}=\alpha$, where $\alpha$ is a real constant, $0<\alpha<\delta$. In these coordinates, the directional derivative along the chord is given by $\frac{\partial}{\partial x_{2}}$. A straightforward computation then gives

$$
\begin{aligned}
u_{k}\left(x_{2}\right) & =\sum_{j=0}^{k} d_{k, 2 j}\left(x_{2}^{2}+\alpha^{2}\right)^{j} \\
\frac{\partial u_{k}}{\partial x_{2}}\left(x_{2}\right) & =\sum_{j=0}^{k} d_{k, 2 j}(2 j) x_{2}\left(x_{2}^{2}+\alpha^{2}\right)^{j-1} .
\end{aligned}
$$

From (4.6)-(4.7), the proof of the theorem follows as it did in the case of onedimensional Brownian motion.

Note that if we restrict exit time moments to a curve given as a circle lying in a plane and centered at the origin, then all moments are constant and the conclusion of Theorem 4.1 fails to hold. It is unclear in general what is the precise relationship between the geometry of the underlying curve to which we restrict, and the corresponding density properties of the exit time moments and their derivatives. 


\section{UNIFORM APPROXIMATION}

We begin by establishing a list of geometric properties of exit time moments for a Brownian motion on the line:

Proposition 5.1. Let $X_{t}$ be a diffusion on $\mathbb{R}$ with infinitesimal generator $\mathcal{L}=$ $\frac{1}{2}\left(a \frac{d}{d x}\right)^{2}$, where $a(x)$ is a smooth positive function. Let I be a compact interval, and define an inner product on continuous functions on $I$ by the formula

$$
\langle f, g\rangle_{a}=\int_{I} f(x) g(x) \frac{1}{a(x)} d x .
$$

Let $v_{k}(x), k$ an integer, be defined as in (1.2)-(1.4). Then, if $j, k>0$,

$$
\begin{aligned}
\left\langle v_{k}, v_{j}\right\rangle_{a} & =\frac{j ! k !}{(j+k) !}\left\langle v_{k+j}, 1\right\rangle_{a}, \\
\left\langle v_{-k}, v_{-j}\right\rangle_{a} & =\frac{2 j}{\gamma^{2}}\left\langle v_{k}, v_{j-1}\right\rangle_{a}, \\
\left\langle v_{k}, v_{-j}\right\rangle_{a} & =0 \\
\left\langle v_{-k}, 1\right\rangle_{a} & =0
\end{aligned}
$$

Proof. Note that the operator $\mathcal{L}$ acting on smooth functions with Dirichlet boundary conditions is selfadjoint with respect to the inner product. Using this fact, integration by parts, the formula (3.5), and induction, the proposition follows easily.

Remark. In higher dimensions, the analogues of the constants $\left\langle v_{k}, 1\right\rangle_{a}$ are closely tied to the underlying geometry of the domain. For example, they completely determine the heat content asymptotics, thus determining a number of geometric invariants $[\mathrm{MM}]$.

Proposition 5.1 indicates that exit time moments do not have good orthogonality properties. We can, nonetheless, give a natural algorithm for producing uniform approximations to continuous functions. We begin by reducing to the case of standard Brownian motion.

If $I$ and $v_{k}$ are as in Proposition 5.1 if $u_{k}$ is the $k$-th moment of the exit time from $I$ of standard Brownian motion on the line, if $\mathcal{M}$ is as in (3.5), and if $\gamma$ is as in (1.1), then a straightforward computation gives

$$
\left\langle u_{k}, u_{j}\right\rangle_{1}=\gamma^{2(j+k)+1}\left\langle v_{k}, v_{j}\right\rangle_{a} .
$$

Thus, for computations involving pairings of the exit time moments, it suffices to understand properties of pairings for the exit time of standard Brownian motion.

For the case of standard Brownian motion on $[-1,1]$ the first exit time moment is given by $u_{1}(x)=\left(1-x^{2}\right)$. We set

$$
U_{m}=\frac{u_{1}^{m}}{\left\langle 1, u_{1}^{m}\right\rangle_{1}}
$$

Let $\eta_{m}^{j}$ be the real constants defined as the coefficients in the expansion

$$
U_{m}=\sum_{j=0}^{m} \eta_{m}^{j} u_{j} .
$$


Theorem 5.2. Suppose that $X_{t}$ is standard Brownian motion on the real line. Let $I=[-1,1]$, let $U_{m}(x)$ be defined by (5.7) and suppose that $\eta_{m}^{j}$ are the constants defined by (5.8). Let $f$ be a continuous function on $[-1,1]$, and let $f_{m}(x)$ be defined by

$$
f_{m}=\sum_{j=0}^{m} \eta_{m}^{j}\left(f * u_{j}\right)
$$

where the product is given by convolution. Then $f_{m}$ converges to $f$ uniformly on $I$.

Proof. From (5.7), $U_{m}(x)$ defines an approximation of unity. Since $f_{m}=f * U_{m}$, it follows that $f_{m}$ converges uniformly to $f$.

It is clear that a similar algorithm holds for general Brownian motions on the line.

Finally, we provide explicit representations of certain special functions in terms of exit time moments. Suppose that $X_{t}$ is standard Brownian motion on the real line, $\delta>0$, and $I=[-\delta, \delta]$. For $s>0$, the unique solution of [2.3) is given by

$$
h(x, s)=\frac{\cosh (\sqrt{2 s} x)}{\cosh (\sqrt{2 s} \delta)} .
$$

From (2.4), we conclude that

$$
\begin{aligned}
& \cosh (\sqrt{2 s} x)=\sum_{k=0}^{\infty}\left(\cosh (\sqrt{2 s} \delta) \frac{(-s)^{k}}{k !}\right) u_{k}(x), \\
& \sinh (\sqrt{2 s} x)=\sum_{k=1}^{\infty}\left(\frac{\cosh (\sqrt{2 s} \delta)}{\sqrt{2 s}} \frac{(-s)^{k}}{k !}\right) u_{-k}(x) .
\end{aligned}
$$

Similarly, let $X_{t}$ be standard Brownian motion on $\mathbb{R}^{n}$, and suppose that $B$ is a ball of radius $\delta$ centered at the origin. Let $\mu=\frac{n-2}{2}$, and set $\nu=(2 s)^{\frac{1}{2}}$. Let $I_{\mu}(x)$ be the Bessel function of pure imaginary part (cf. [WW]):

$$
I_{\mu}(x)=\sum_{r=0}^{\infty} \frac{\left(\frac{1}{2} x\right)^{\mu+2 r}}{r !(r+\mu) !} .
$$

It is a theorem of Doob D] (cf. W] for closely related work) that

$$
\mathbb{E}^{x}\left[e^{-s \tau}\right]=\left(\frac{\delta}{x}\right)^{\mu} I_{\mu}(\nu x) / I_{\mu}(\nu \delta) .
$$

From (2.4), (5.13), and (5.14) we see that

$$
I_{\mu}(\nu x)=\left(\frac{x}{\delta}\right)^{\mu} \sum_{k=0}^{\infty}\left(I_{\mu}(\nu \delta) \frac{(-s)^{k}}{k !}\right) u_{k}(x) .
$$

In particular, we have proven

Proposition 5.3. Suppose that $X_{t}$ is Brownian motion on $\mathbb{R}^{2}$. Suppose that $B$ is $a$ disk of radius $\delta$ centered at the origin and that $u_{k}(x)$ are the exit time moments of $X_{t}$ from $B$ as in (2.7). Let $\nu=(2 s)^{\frac{1}{2}}$. Then

$$
I_{0}(\nu x)=\sum_{k=0}^{\infty}\left(I_{0}(\nu \delta) \frac{(-s)^{k}}{k !}\right) u_{k}(x)
$$




\section{REFERENCES}

[D] J. L. Doob, A probability approach to the heat equation, Trans. Amer. Math. Soc. 80, 216-280 (1955). MR 18:76g

[Ha] R. Z. Ha'sminskii, Probabilistic representations of the solutions of some differential equations, In: Proc. 6th All Union Conf. on Theor. Prob. and Math. Stat. (Vilnius 1960) (1960).

$[\mathrm{KMM}]$ K. Kinateder, P. McDonald, and D. Miller, Exit time moments, boundary value problems and the geometry of domains in Euclidean space, Prob. Theory and Related Fields 111, 469-487 (1998). MR 99h:60151

[MM] P. McDonald and R. Meyers, Dirichlet spectrum and heat content, Jour. Funct. Anal. 200, 150-159 (2003). MR 2004c:58074

[W] J. G. Wendel, Hitting spheres with Brownian motion, Ann. Probability 8, 164-169 (1980). MR 80m:60085

[WW] E. T. Whittaker and G. N. Watson, A Course of Modern Analysis, fourth edition, Cambridge University Press, London, 1940. MR 31:2375

Department of Statistics, Columbia University, New York, New York 10027

E-mail address: vp@stat.columbia.edu

Department of Mathematics, New College of Florida, Sarasota, Florida 34243

E-mail address: ptm@virtu.sar.usf.edu 